\title{
New Exact Solutions for High Dispersive Cubic-Quintic Nonlinear Schrödinger Equation
}

\author{
Yongan Xie and Shengqiang Tang \\ School of Mathematics and Computing Science, Guilin University of Electronic Technology, Guilin, Guangxi 541004, China \\ Correspondence should be addressed to Shengqiang Tang; tangsq@guet.edu.cn
}

Received 6 May 2014; Revised 8 July 2014; Accepted 18 July 2014; Published 5 August 2014

Academic Editor: Sheng Zhang

Copyright (C) 2014 Y. Xie and S. Tang. This is an open access article distributed under the Creative Commons Attribution License, which permits unrestricted use, distribution, and reproduction in any medium, provided the original work is properly cited.

We study a class of high dispersive cubic-quintic nonlinear Schrödinger equations, which describes the propagation of femtosecond light pulses in a medium that exhibits a parabolic nonlinearity law. Applying bifurcation theory of dynamical systems and the Fan sub-equations method, more types of exact solutions, particularly solitary wave solutions, are obtained for the first time.

\section{Introduction}

Propagation of short pulses in optical fibers is governed by the well-known nonlinear Schrödinger equation (NLS) [1]. In recent years, There have been extensive study and application of NLS. The main purpose of this paper is to discuss the traveling wave solutions for a class of high dispersive cubic-Quintic nonlinear Schrödinger equations describing the ultrashort light pulse propagation as in the following:

$$
E_{z}=-i \frac{\beta_{2}}{2} E_{t t}+i \gamma_{1}|E|^{2} E+\frac{\beta_{3}}{6} E_{t t t}+i \frac{\beta_{4}}{24} E_{t t t t}-i \gamma_{2}|E|^{4} E
$$

where $E(z, t)$ is the slowly varying envelope of the electric field, $\beta_{2}$ is the parameter of the group velocity dispersion, $\beta_{3}$ and $\beta_{4}$ are, respectively, the third-order and fourth-order dispersions, and $\gamma_{1}$ and $\gamma_{2}$ are the nonlinearity coefficients. When the higher order terms are ignored, we obtain the NLS. However, for femtosecond light pulses, whose duration is shorter than $10 \mathrm{fs}$, the last three terms are not ignored. Equation (1) was derived by Palacios and Fernández-Diáz [2]. Azzouzi et al. [3] by using the extended hyperbolic auxiliary equation method in getting the exact explicit solutions to (1). He et al. [4] find the exact bright, dark, and gray analytical nonautonomous soliton solutions of the generalized CQNLSE with spatially inhomogeneous group velocity dispersion (GVD) and amplification or attenuation by the similarity transformation method under certain parametric conditions.

We will study (1) by using the improved Fan subequation method. As a result, more types of exact solutions to (1) are obtained, which include solitons, kink solutions, and Jacobian elliptic function solutions with double periods. The rest of this paper is organized as follows. In Section 2, we give the mathematical framework of the improved method. In Section 3, we apply it to the generalized equation (1) for finding more exact solutions. Finally, some conclusions are given.

\section{The Ansatz Solution and Fan Subequation Method}

The integrability of a nonlinear equation can be studied by applying the Painleve analysis. It is widely believed that possession of the Painleve property is a sufficient criterion for integrability. Moreover, there exists another technique which basically consists of expressing the solution in terms of an amplitude and a phase function as an approach to find exact solutions of nonlinear evolution equations. We will make use of this formalism looking for exact solution of (1) such as

$$
E(z, t)=e^{i\left(w_{0} z-w t\right)} \varphi(\xi),
$$


where $\varphi(\xi)$ is a real fuction and $\xi=v_{0} z-v t$. By inserting the expressions (2) into (1), and separating real and imaginary parts, we obtain

$$
\begin{gathered}
l_{1} \varphi^{\prime}(\xi)+l_{3} \varphi^{\prime \prime \prime}(\xi)=0, \\
l_{0} \varphi(\xi)+l_{2} \varphi^{\prime \prime}(\xi)+l_{4} \varphi^{\prime \prime \prime \prime}(\xi)+\gamma_{1} \varphi^{3}(\xi)-\gamma_{2} \varphi^{5}(\xi)=0
\end{gathered}
$$

where

$$
\begin{aligned}
& l_{0}=\frac{1}{2} w^{2}\left(\beta_{2}+\frac{1}{3} \beta_{3} w+\frac{1}{12} \beta_{4} w^{2}\right)-w_{0}, \\
& l_{1}=w v\left(\beta_{2}+\frac{1}{2} \beta_{3} w+\frac{1}{6} \beta_{4} w^{2}\right)-v_{0}, \\
& l_{2}=-v^{2}\left(\frac{1}{2} \beta_{2}+\frac{1}{2} \beta_{3} w+\frac{1}{4} \beta_{4} w^{2}\right), \\
& l_{3}=-\frac{1}{6}\left(\beta_{3}+\beta_{4} w\right) v^{3}, \quad l_{4}=\frac{1}{24} \beta_{4} v^{4} .
\end{aligned}
$$

Let $l_{1}=l_{3}=0$, and we get

$$
\begin{gathered}
w=-\frac{\beta_{3}}{\beta_{4}}, \quad v_{0}=\frac{v \beta_{3}\left(\beta_{3}^{2}-3 \beta_{2} \beta_{4}\right)}{3 \beta_{4}^{2}}, \\
l_{0}=-w_{0}-\frac{\beta_{3}^{2}\left(\beta_{3}^{2}-4 \beta_{2} \beta_{4}\right)}{8 \beta_{4}^{3}}, \quad l_{2}=\frac{v^{2}\left(\beta_{3}^{2}-2 \beta_{2} \beta_{4}\right)}{4 \beta_{4}}, \\
l_{4}=\frac{1}{24} \beta_{4} v^{4} .
\end{gathered}
$$

Then, (3a) and (3b) become

$$
l_{0} \varphi(\xi)+l_{2} \varphi^{\prime \prime}(\xi)+l_{4} \varphi^{\prime \prime \prime \prime}(\xi)+\gamma_{1} \varphi^{3}(\xi)-\gamma_{2} \varphi^{5}(\xi)=0 .
$$

We introduce auxiliary equation:

$$
\varphi^{\prime}(\xi)=\epsilon \sqrt{c_{0}+c_{1} \varphi(\xi)+c_{2} \varphi^{2}(\xi)+c_{3} \varphi^{3}(\xi)+c_{4} \varphi^{4}(\xi)},
$$

where $\epsilon= \pm 1$, which is known as Fan subequation method and proposed by Fan in [5]. This method is proposed to seek more types of exact solutions of nonlinear partial differential equations. Obviously, (7) is equivalent to the twodimensional systems as follows:

$$
\frac{d \varphi}{d \xi}=y, \quad \frac{d y}{d \xi}=\frac{1}{2}\left(c_{1}+2 c_{2} \varphi+3 c_{3} \varphi^{2}+4 c_{4} \varphi^{3}\right),
$$

which has the Hamiltonian function:

$$
H(\varphi, y)=y^{2}-\left(c_{1} \varphi+c_{2} \varphi^{2}+c_{3} \varphi^{3}+c_{4} \varphi^{4}\right)=c_{0} .
$$

One can easily find that $c_{0}$ corresponds to the Hamiltonian constant and (7) is equivalent to the Hamiltonian system (8). Thus, in order to search the exact solutions of (7) we need only to discuss (8). For a fixed $c_{0}$, (9) determines a set of orbits of (8). As $c_{0}$ varies, (9) defines different families of orbits of (8) which have different dynamical behavior. Below we will first study the bifurcation of phase portraits of (8) by making use of bifurcation method of dynamical systems and with the aid of the computer symbolic system Mathematica. Then according to the obtained bifurcation and the Hamiltonian function (9), we will gain many new exact solutions of (7) for all possible parameters $c_{j}[6]$.

Substituting (7) into (6), we have

$$
\begin{aligned}
& \left(-\gamma_{2}+24 l_{4} c_{4}^{2}\right) \varphi^{5}+30 l_{4} c_{3} c_{4} \varphi^{4} \\
& +\left(2 l_{2} c_{4}+l_{4}\left(20 c_{2} c_{4}+\frac{15}{2} c_{3}^{2}\right)+\gamma_{1}\right) \varphi^{3} \\
& +\left(\frac{3}{2} l_{2} c_{3}+l_{4}\left(\frac{15}{2} c_{2} c_{3}+1_{5} c_{1} c_{4}\right)\right) \varphi^{2} \\
& +\left(l_{0}+l_{2} c_{2}+l_{4}\left(\frac{9}{2} c_{1} c_{3}+1_{2} c_{0} c_{4}+c_{2}^{2}\right)\right) \varphi \\
& +\frac{1}{2} l_{2} c_{1}+l_{4}\left(3 c_{0} c_{3}+\frac{1}{2} c_{1} c_{2}\right)=0 .
\end{aligned}
$$

Setting all the coefficients of $\varphi^{i}(i=0,1, \ldots, 5)$ to zero, and solving the obtained algebraic equations, we find the following sets of solutions (I)

$$
\begin{aligned}
& c_{1}= c_{3}=0 \\
& c_{2}= \frac{1}{v^{2}}\left(-\frac{3\left(\beta_{3}^{2}-2 \beta_{2} \beta_{4}\right)}{5 \beta_{4}^{2}}-\frac{6 \gamma_{1} \sqrt{\gamma_{2} / \beta_{4}}}{5 \gamma_{2}}\right), \\
& c_{4}= \frac{1}{v^{2}} \sqrt{\frac{\gamma_{2}}{\beta_{4}}}, \\
& c_{0}=\left(\left(50 w_{0} \gamma_{2} \beta_{4}^{3}-3 \gamma_{1}^{2} \beta_{4}^{3}-52 \gamma_{2} \beta_{2} \beta_{3}^{2} \beta_{4}\right.\right. \\
&\left.\quad+27 \gamma_{2} \beta_{2}^{2} \beta_{4}^{2}+13 \gamma_{2} \beta_{3}^{4}\right) \\
&\left.\quad \times \sqrt{\frac{\gamma_{2}}{\beta_{4}}}+12 \gamma_{1} \gamma_{2} \beta_{4}\left(\beta_{3}^{2}-2 \beta_{2} \beta_{4}\right)\right) \\
& \quad \times\left(25 \gamma_{2}^{2} \beta_{4}^{3} v^{2}\right)^{-1}
\end{aligned}
$$

and (II)

$$
\begin{aligned}
& c_{1}=c_{3}=0, \\
& c_{2}=\frac{1}{5 v^{2}}\left(-\frac{3\left(\beta_{3}^{2}-2 \beta_{2} \beta_{4}\right)}{\beta_{4}^{2}}+\frac{6 \gamma_{1} \sqrt{\gamma_{2} / \beta_{4}}}{\gamma_{2}}\right), \\
& c_{4}=-\frac{1}{v^{2}} \sqrt{\frac{\gamma_{2}}{\beta_{4}}}
\end{aligned}
$$




$$
\begin{aligned}
c_{0}=( & -\left(50 w_{0} \gamma_{2} \beta_{4}^{3}-3 \gamma_{1}^{2} \beta_{4}^{3}-52 \gamma_{2} \beta_{2} \beta_{3}^{2} \beta_{4}\right. \\
& \left.+27 \gamma_{2} \beta_{2}^{2} \beta_{4}^{2}+13 \gamma_{2} \beta_{3}^{4}\right) \\
& \left.\times \sqrt{\frac{\gamma_{2}}{\beta_{4}}}+12 \gamma_{1} \gamma_{2} \beta_{4}\left(\beta_{3}^{2}-2 \beta_{2} \beta_{4}\right)\right) \\
& \times\left(25 \gamma_{2}^{2} \beta_{4}^{3} v^{2}\right)^{-1}
\end{aligned}
$$

and (III), $\gamma_{2}=0$ :

$$
\begin{aligned}
c_{0}= & \left(\left(-\beta_{3}^{2}+2 \beta_{2} \beta_{4}\right)\right. \\
& \left.\times\left(200 \beta_{4}^{3} w_{0}+73 \beta_{3}^{4}-292 \beta_{2} \beta_{3}^{2} \beta_{4}+192 \beta_{2}^{2} \beta_{4}^{2}\right)\right) \\
& \times\left(150 \beta_{4}^{5} \gamma_{1} v^{2}\right)^{-1}, \\
c_{1}= & \frac{200 \beta_{4}^{3} w_{0}+73 \beta_{3}^{4}-292 \beta_{2} \beta_{3}^{2} \beta_{4}+192 \beta_{2}^{2} \beta_{4}^{2}}{150 \beta_{4}^{3} \gamma_{1} v^{2}} \\
\times & \sqrt{\frac{-5 \gamma_{1}}{\beta_{4}}}, \\
c_{2}= & \frac{6\left(-\beta_{3}^{2}+2 \beta_{2} \beta_{4}\right)}{5 \beta_{4}^{2} v^{2}}, \quad c_{3}= \pm \frac{4}{5 v^{2}} \sqrt{\frac{-5 \gamma_{1}}{\beta_{4}}}, \\
c_{4}= & 0,
\end{aligned}
$$

where $w_{0}, v$ are any real number and $v \neq 0$.

\section{Exact Solutions of High Dispersive Cubic- Quintic Nonlinear Schrödinger Equation}

3.1. Case (I) and Case (II). In this case, the Hamiltonian system (8) becomes

$$
\frac{d \varphi}{d \xi}=y, \quad \frac{d y}{d \xi}=c_{2} \varphi+2 c_{4} \varphi^{3},
$$

and the Hamiltonian function (9) reduces to

$$
H_{1}(\varphi, y)=y^{2}-c_{2} \varphi^{2}-c_{4} \varphi^{4}=c_{0} .
$$

Now we discuss the bifurcations of phase portraits of (14). Obviously all the equilibrium points of (14) lie in the $\varphi$-axis and their abscissas are the real zeros of $f(\varphi)=c_{2} \varphi+2 c_{4} \phi^{3}$. Thus, we have the following proposition on the distribution of the equilibrium points of (14).

\section{Proposition 1.}

(1) For $c_{2} c_{4}<0$, (14) has three equilibria points at $E_{10}(0,0), E_{11}\left(\psi_{11}, 0\right)$, and $E_{12}\left(\psi_{12}, 0\right)$, where $\psi_{11}=$ $-\sqrt{-c_{2} /\left(2 c_{4}\right)}, \psi_{12}=\sqrt{-c_{2} /\left(2 c_{4}\right)}$.

(2) For $c_{2} c_{4} \geq 0$, (14) has a unique equilibrium at $E_{10}(0,0)$.
Using the bifurcation theory of dynamical systems [7-9], the $\left(c_{2}, c_{4}\right)$-plane was divided into four subregions:

$$
\begin{array}{ll}
D 1:\left\{c_{2}>0, c_{4}>0\right\} ; & D 2:\left\{c_{2}<0, c_{4}>0\right\} ; \\
D 3:\left\{c_{2}<0, c_{4}<0\right\} ; & D 4:\left\{c_{2}>0, c_{4}<0\right\} .
\end{array}
$$

The phase portraits of (14) are shown in Figure 1.

For the function defined by (15), we have

$$
\begin{aligned}
& h_{10}=H_{1}\left(\psi_{10}, 0\right)=0, \\
& h_{11}=h_{12}=H_{1}\left(\psi_{11}, 0\right)=H_{1}\left(\psi_{12}, 0\right)=\frac{c_{2}^{2}}{4 c_{4}} .
\end{aligned}
$$

Let $M\left(w_{e}, z_{e}\right)$ be the coefficient matrix of the system (14) at an equilibrium point $\left(w_{e}, z_{e}\right)$. Then, we have

$$
J\left(w_{e}, 0\right)=\operatorname{det}\left(M\left(w_{e}, 0\right)\right)=-\left(c_{2}+6 c_{4} w_{e}^{2}\right) .
$$

By the theory of planar dynamical systems, we know that for an equilibrium point of a planar integrable system, if $J<0$, then the equilibrium point is a saddle point; if $J>0$ and Trace $\left(M\left(w_{e}\right)\right)=0$, then it is a center point; if $J>0$ and $\left(\operatorname{Trace}\left(M\left(w_{e}, z_{e}\right)\right)\right)^{2}-4 J\left(w_{e}, z_{e}\right)>0$, then it is a node; if $J=0$ and the index of the equilibrium point is 0 , then it is a cusp; otherwise, it is a high order equilibrium point.

Below, we will give explicit and exact solutions of (7) (also (6)). We always let $\Delta=c_{2}^{2}-4 c_{0} c_{4}$ and only pay attention to the bounded solutions of (6).

(1) Suppose that $\left(c_{2}, c_{4}\right) \in D 4$.

(a) For $c_{0} \in\left(c_{2}^{2} /\left(4 c_{4}\right), 0\right),(7)$ has periodic solutions:

$$
\varphi_{ \pm 1}= \pm \sqrt{\frac{c_{2}+\sqrt{\Delta}}{-2 c_{4}}+\frac{\sqrt{\Delta}}{c_{4}} \mathrm{sn}^{2}\left(\sqrt{\frac{c_{2}+\sqrt{\Delta}}{2}} \xi, \sqrt{\frac{2 \sqrt{\Delta}}{c_{2}+\sqrt{\Delta}}}\right)},
$$

where $\operatorname{sn}(x, k)$ and below $\operatorname{cn}(x, k)$ are Jacobian elliptic functions with modulus $k$ [10]. The profiles of periodic solutions are shown in Figure 2.

Thus, we obtain the following solutions of (1):

$$
\begin{aligned}
& E(z, t) \\
& = \pm e^{i\left(w_{0} z+\beta_{3} t / \beta_{4}\right)} \\
& \quad \times \sqrt{\frac{c_{2}+\sqrt{\Delta}}{-2 c_{4}}+\frac{\sqrt{\Delta}}{c_{4}} \operatorname{sn}^{2}\left(\sqrt{\frac{c_{2}+\sqrt{\Delta}}{2}} \xi, \sqrt{\frac{2 \sqrt{\Delta}}{c_{2}+\sqrt{\Delta}}}\right) .} .
\end{aligned}
$$

(b) For $c_{0}=0$, we have solitary wave solutions of (7):

$$
\varphi_{ \pm 2}= \pm \sqrt{-\frac{c_{2}}{c_{4}}} \operatorname{sech}\left(\sqrt{c_{2}} \xi\right) .
$$

Thus, we obtain the following solutions of (1):

$$
E(z, t)= \pm e^{i\left(w_{0} z+\beta_{3} t / \beta_{4}\right)} \sqrt{-\frac{c_{2}}{c_{4}}} \operatorname{sech}\left(\sqrt{c_{2}} \xi\right) .
$$

The profiles of solutions of (22) are shown in Figure 3. 


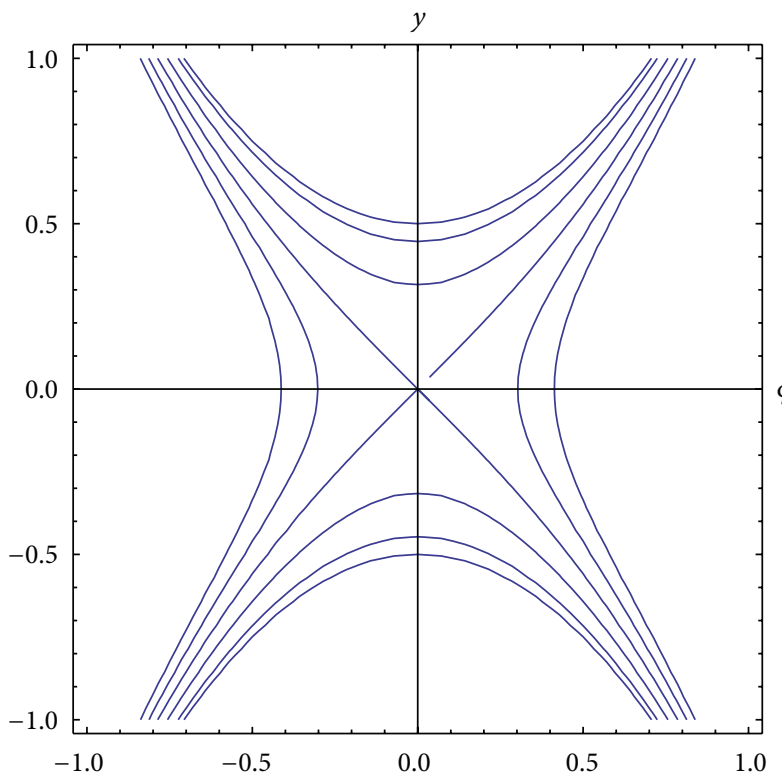

(a) $\left(c_{2}, c_{4}\right) \in D 1$

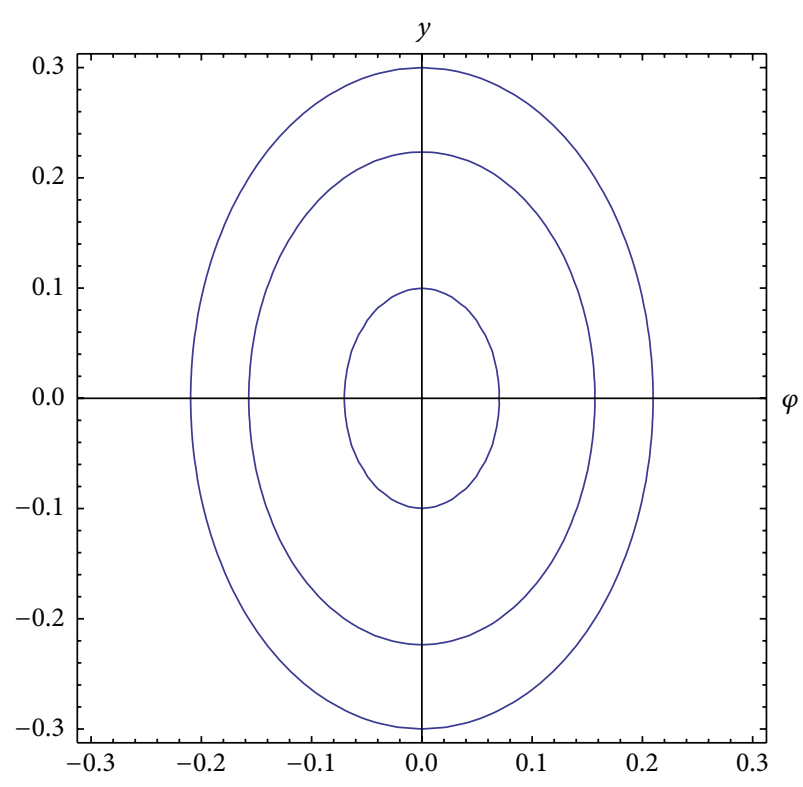

(c) $\left(c_{2}, c_{4}\right) \in D 3$

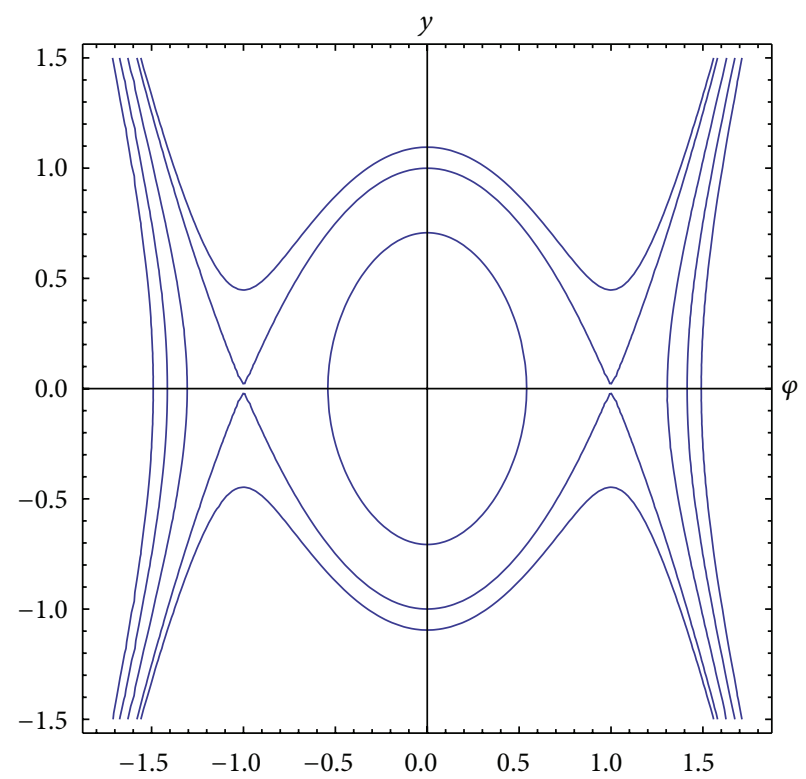

(b) $\left(c_{2}, c_{4}\right) \in D 2$

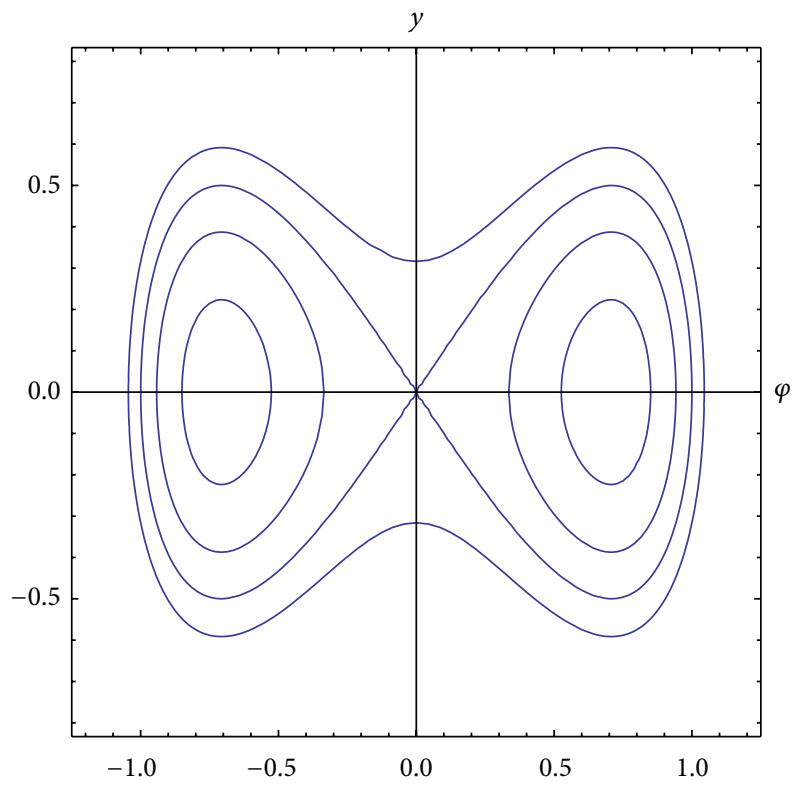

(d) $\left(c_{2}, c_{4}\right) \in D 4$

FIGURE 1: The phase portraits of system (14).

Remark 2. By the expression of $c_{0}$, there always exists a $w_{0}$ such that $c_{0}=0$ if $\left(c_{2}, c_{4}\right) \in D 4$.

Because of the limitation of length, we omit the expression of $E(z, t)$, beginning from here.

(c) For $c_{0}>0$, (6) has periodic solution:

$$
\varphi_{3}=\sqrt{\frac{c_{2}+\sqrt{\Delta}}{-2 c_{4}}} \operatorname{cn}\left(\sqrt[4]{\Delta} \xi, \sqrt{\frac{c_{2}+\sqrt{\Delta}}{2 \sqrt{\Delta}}}\right) .
$$

(2) Suppose that $\left(c_{2}, c_{4}\right) \in D 2$. (a) For $c_{0} \in\left(0, c_{2}^{2} /\left(4 c_{4}\right)\right)$, system (14) has periodic solution:

$\varphi_{ \pm 4}= \pm \sqrt{\frac{-c_{2}-\sqrt{\Delta}}{-c_{2}+\sqrt{\Delta}}} \operatorname{sn}\left(\sqrt{\frac{-c_{2}+\sqrt{\Delta}}{2}} \xi, \sqrt{\frac{-c_{2}-\sqrt{\Delta}}{-c_{2}+\sqrt{\Delta}}}\right)$.

(b) For $c_{0}=c_{2}^{2} /\left(4 c_{4}\right)$, there exists smooth kink wave solution of (14) as follows:

$$
\varphi_{ \pm 5}= \pm \sqrt{\frac{-c_{2}}{2 c_{4}}} \tanh \left(\sqrt{\frac{-c_{2}}{2}} \xi\right) .
$$




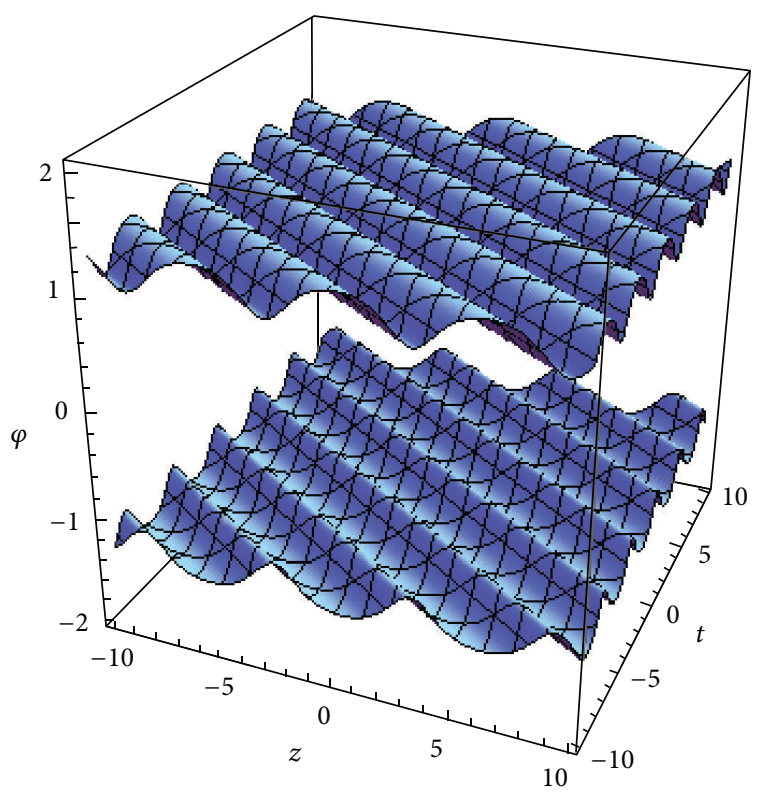

(a) The 3D wave profiles of solutions $\varphi_{ \pm 1}$

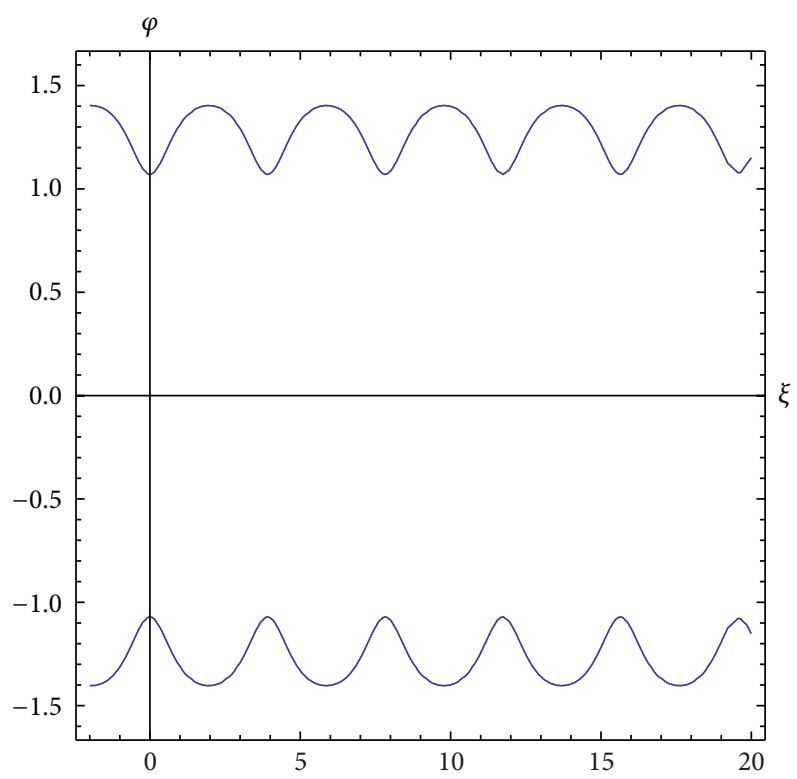

(b) The 2D wave profiles of solutions $\varphi_{ \pm 1}$ for $t=0$

FIGURE 2: The wave profiles of solutions $\varphi_{ \pm 1}$ with the parameters values: $\gamma_{1}=\gamma_{2}=\beta_{1}=\beta_{2}=\beta_{3}=v=1, w_{0}=0.38$

(3) If $\left(c_{2}, c_{4}\right) \in D_{3}$, for $c_{0}>0$, we have the following periodic solution of (14):

$$
\varphi_{ \pm 6}= \pm \sqrt{\frac{-c_{2}-\sqrt{\Delta}}{2 c_{4}}} \operatorname{cn}\left(\sqrt[4]{\Delta} \xi, \sqrt{\frac{c_{2}+\sqrt{\Delta}}{2 \sqrt{\Delta}}}\right) .
$$

(4) If $\left(c_{2}, c_{4}\right) \in D_{1}$, it is observed from Figure 1(a) that there is no bounded solution of system (14).

3.2. The Case (III). For this case, the Hamiltonian system (8) becomes

$$
\frac{d \varphi}{d \xi}=y, \quad \frac{d y}{d \xi}=f(\varphi)=\frac{1}{2}\left(c_{1}+2 c_{2} \varphi+3 c_{3} \varphi^{2}\right)
$$

and the Hamiltonian function (9) reduces to

$$
H_{1}(\varphi, y)=y^{2}-c_{1} \varphi-c_{2} \varphi^{2}-c_{3} \varphi^{3}=c_{0} .
$$

Similar to the previous discussion, we have the following proposition.

\section{Proposition 3.}

(1) For $c_{2}^{2}-3 c_{1} c_{3}>0$, (27) has two equilibria at $E_{21}\left(\psi_{21}, 0\right)$ and $E_{22}\left(\psi_{22}, 0\right)$, where

$$
\psi_{21}=\frac{-c_{2}-\sqrt{c_{2}^{2}-3 c_{1} c_{3}}}{3 c_{3}}, \quad \psi_{22}=\frac{-c_{2}+\sqrt{c_{2}^{2}-3 c_{1} c_{3}}}{3 c_{3}} .
$$

(2) For $c_{2}^{2}-3 c_{1} c_{3}=0$, (27) has a unique equilibrium at $E_{20}\left(\psi_{20}, 0\right)$, where $\psi_{20}=-c_{2} /\left(3 c_{3}\right)$.
(3) For $c_{2}^{2}-3 c_{1} c_{3}<0$, (27) has no equilibrium.

Let $h_{2 i}=H_{1}\left(\psi_{2 i}, 0\right), i=0,1,2$, and notice that we need only to consider the case $c_{3} \geq 0$ because of the invariance of (27) under the transformations $\phi \rightarrow-\phi, y \rightarrow-y$, and $c_{3} \rightarrow-c_{3}$.

(1) $c_{2}^{2}-3 c_{1} c_{3}>0$ and $c_{0} \in\left(h_{21}, h_{22}\right)$. In this case,

$$
c_{3} \varphi^{3}+c_{2} \varphi^{2}+c_{1} \varphi+c_{0}=0
$$

has three mutually different real roots $\varphi_{m}<\varphi_{l}<\varphi_{M}$; thus,

$$
c_{3} \varphi^{3}+c_{2} \varphi^{2}+c_{1} \varphi+c_{0}=c_{3}\left(\varphi-\varphi_{m}\right)\left(\varphi-\varphi_{l}\right)\left(\varphi-\varphi_{M}\right) .
$$

Equation (7) has periodic wave solutions as follows:

$$
\begin{aligned}
& \varphi_{8}= \varphi_{M}-\left(\left(\varphi_{M}-\varphi_{l}\right)\left(\varphi_{M}-\varphi_{m}\right)\right) \\
& \times\left(\varphi_{M}-\varphi_{m}-\left(\varphi_{l}-\varphi_{m}\right) \mathrm{sn}^{2}\right. \\
&\left.\times\left(\frac{\sqrt{c_{3}\left(\varphi_{M}-\varphi_{m}\right)}}{2} \xi \sqrt{\frac{\varphi_{l}-\varphi_{m}}{\varphi_{M}-\varphi_{m}}}\right)\right)^{-1}, \\
& \varphi_{9}=\varphi_{m}+\left(\varphi_{l}-\varphi_{m}\right) \operatorname{sn}^{2}\left(\frac{\sqrt{c_{3}\left(\varphi_{M}-\varphi_{m}\right)}}{2} \xi \sqrt{\frac{\varphi_{l}-\varphi_{m}}{\varphi_{M}-\varphi_{m}}}\right) .
\end{aligned}
$$

(2) $c_{2}^{2}-3 c_{1} c_{3}>0$ and $c_{0}=h_{22}$. In this case, $\varphi_{22}$ is double root of (30); suppose that $\varphi_{t}$ is other root of the equation, 


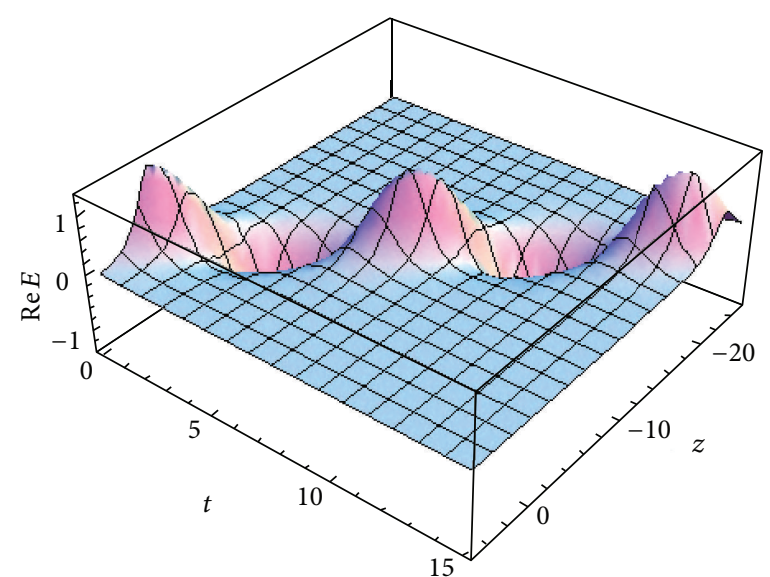

(a) The 3D wave profile of real part

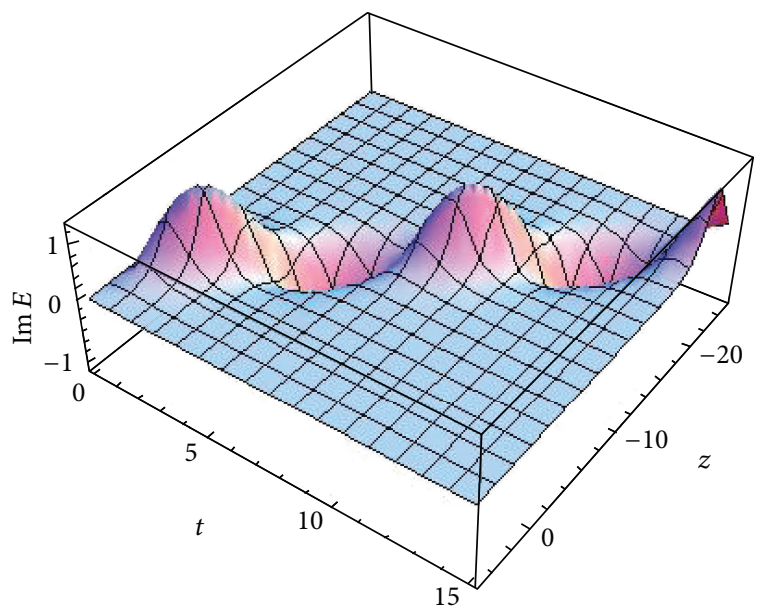

(c) The 3D wave profile of imaginary part

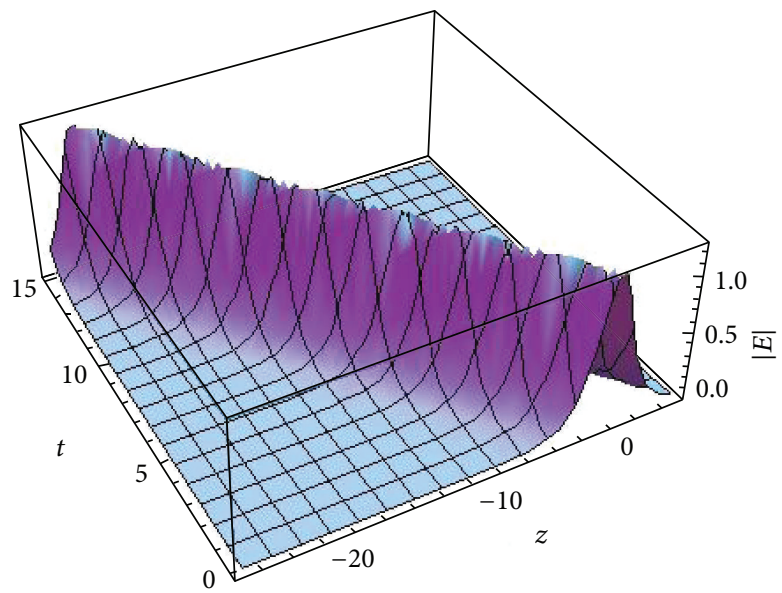

(e) The $3 \mathrm{D}$ wave profile of $|E|$

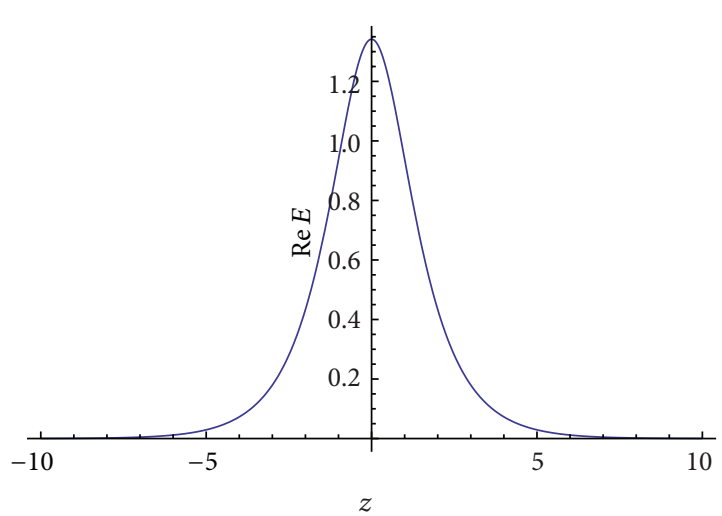

(b) The 2D wave profile of real part

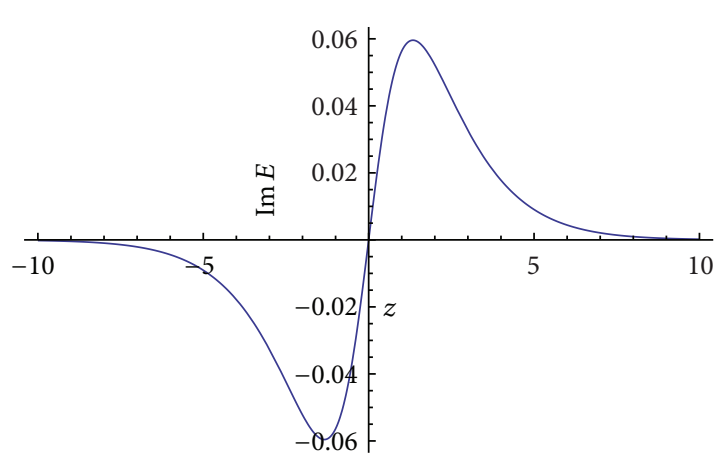

(d) The 2D wave profile of imaginary part

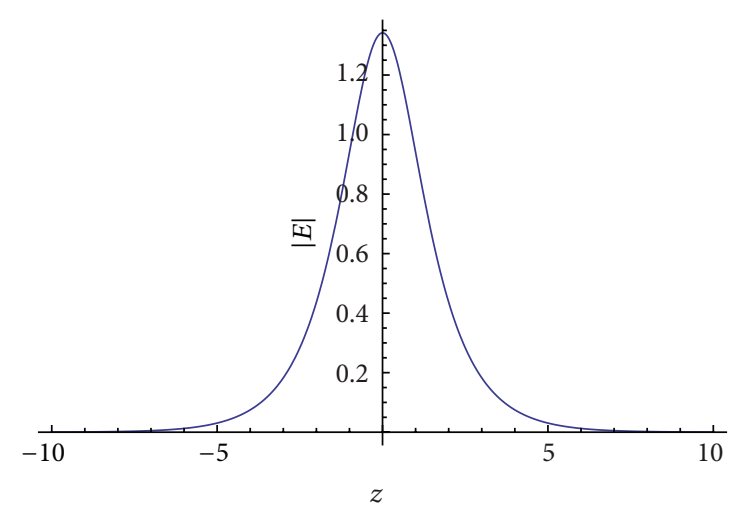

(f) The 2D wave profile of $|E|$

FIGURE 3: The wave profile of solutions (22), with the parameters values: $\gamma_{1}=\gamma_{2}=\beta_{1}=\beta_{2}=\beta_{3}=v=1, w_{0}=0.06$.

obviously $\varphi_{t}<\varphi_{22}$, and we have a solitary wave solution of peak type of (7) as follows:

$$
\varphi_{10}=\varphi_{t}+\left(\varphi_{22}-\varphi_{t}\right) \tanh ^{2}\left(\frac{1}{2} \sqrt{c_{3}\left(\varphi_{22}-\varphi_{t}\right)} \xi\right) .
$$

\section{Conclusions}

In this study, we apply bifurcation theory of dynamical systems and the Fan subequation method to investigate (1), and many new exact solutions have been obtained; most 
importantly, under more general conditions than [3], to the best of our knowledge, these solutions have not been reported in the literature. This method can help us find exact solutions of other types of nonlinear dispersion partial differential equations.

\section{Conflict of Interests}

The authors declare that there is no conflict of interests regarding the publication of this paper.

\section{Acknowledgments}

This work was supported by the National Natural Science Foundation of China (11261013 and 11361017), Natural Science Foundation of Guangxi (2012GXNSFAA053003 and 2013GXNSFAA019010), and the Foundation of Guangxi Key Lab of Trusted Software.

\section{References}

[1] G. P. Agrawal and C. Headley III, "Kink solitons and optical shocks in dispersive nonlinear media," Physical Review A, vol. 46, no. 3, pp. 1573-1577, 1992.

[2] S. L. Palacios and J. M. Fernández-Díaz, "Black optical solitons for media with parabolic nonlinearity law in the presence of fourth order dispersion," Optics Communications, vol. 178, no. 4, pp. 457-460, 2000.

[3] F. Azzouzi, H. Triki, K. Mezghiche, and A. El Akrmi, "Solitary wave solutions for high dispersive cubic-quintic nonlinear Schrödinger equation," Chaos, Solitons and Fractals, vol. 39, no. 3, pp. 1304-1307, 2009.

[4] J. He, J. Zhang, M. Zhang, and C. Dai, "Analytical nonautonomous soliton solutions for the cubic-quintic nonlinear Schrödinger equation with distributed coefficients," Optics Communications, vol. 285, no. 5, pp. 755-760, 2012.

[5] E. Fan, "Uniformly constructing a series of explicit exact solutions to nonlinear equations in mathematical physics," Chaos, Solitons \& Fractals, vol. 16, no. 5, pp. 819-839, 2003.

[6] Y. Xie, S. Tang, and D. Feng, "New exact solutions to the generalized KdV equation with generalized evolution," Pramana, vol. 78, no. 4, pp. 499-511, 2012.

[7] J. Guckenheimer and P. Holmes, Nonlinear Oscillations, Dynamical Systems, and Bifurcations of Vector Fields, Springer, New York, NY, USA, 1983.

[8] D. Luo, Bifurcation Theory and Methods of Dynamical Systems, World Scientific, Singapore, 1997.

[9] P. Lawrence, Differential Equations and Dynamical Systems, Springer, New york, NY, USA, 1991.

[10] P. F. Byrd and M. D. Friedman, Handbook of Elliptic Integrals for Engineers and Physicists, Springer, Berlin, Germany, 1954. 